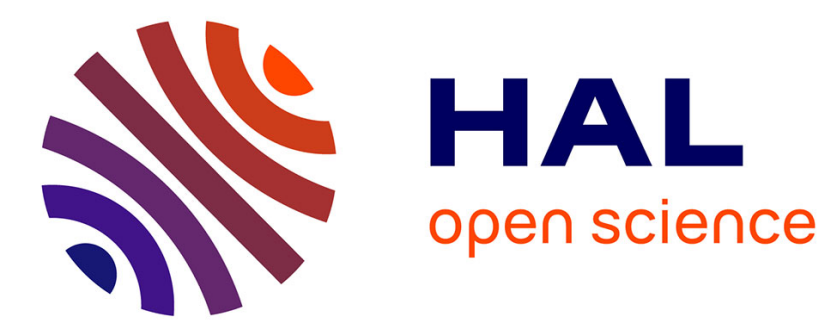

\title{
Scheduling for fault-tolerance: an introduction
}

\author{
Guillaume Aupy, Yves Robert
}

\section{To cite this version:}

Guillaume Aupy, Yves Robert. Scheduling for fault-tolerance: an introduction. [Research Report] RR-8971, INRIA. 2016. hal-01393192v2

\section{HAL Id: hal-01393192 \\ https://hal.inria.fr/hal-01393192v2}

Submitted on 13 Dec 2016

HAL is a multi-disciplinary open access archive for the deposit and dissemination of scientific research documents, whether they are published or not. The documents may come from teaching and research institutions in France or abroad, or from public or private research centers.
L'archive ouverte pluridisciplinaire HAL, est destinée au dépôt et à la diffusion de documents scientifiques de niveau recherche, publiés ou non, émanant des établissements d'enseignement et de recherche français ou étrangers, des laboratoires publics ou privés. 


\section{Scheduling for}

fault-tolerance: an

\section{introduction}

Guillaume Aupy, Yves Robert

RESEARCH

REPORT

$\mathbf{N}^{\circ} 8971$

November 2016

Project-Team ROMA 



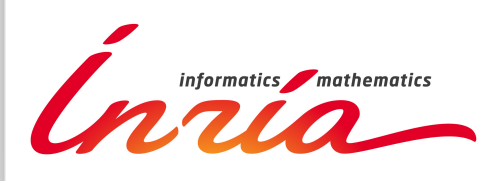

\title{
Scheduling for fault-tolerance: an introduction
}

\author{
Guillaume Aupy*, Yves Robert ${ }^{\dagger \ddagger}$ \\ Project-Team ROMA \\ Research Report $n^{\circ} 8971$ - November 2016 - 28 pages
}

\begin{abstract}
This report provides an introduction to the design of scheduling algorithms to cope with faults on large-scale parallel platforms. We study checkpointing and show how to derive the optimal checkpointing period. Then we explain how to combine checkpointing with fault prediction, and discuss how the optimal period is modified when this combination is used. Finally we follow the very same approach for the combination of checkpointing with replication.
\end{abstract}

Key-words: scheduling, fault, checkpoint, prediction, replication.

* Vanderbilt University, USA

† University of Tennessee, Knoxville TN, USA

¥ Equipe-projet ROMA, Laboratoire LIP, École Normale Supérieure de Lyon \& Inria, France

RESEARCH CENTRE

GRENOBLE - RHÔNE-ALPES

Inovallée

655 avenue de l'Europe Montbonnot

38334 Saint Ismier Cedex 


\section{Ordonnancement en présence de pannes: une introduction}

Résumé : Ce rapport propose une introduction aux techniques d'ordonnancement pour les applications divisibles déployées à grande échelle et confrontées à des pannes au cours de l'exécution. Nous étudions les techniques de checkpoint, et montrons comment obtenir la période de checkpoint optimale. Nous montrons ensuite comment combiner le checkpoint avec la prédiction de fautes, puis avec la réplication.

Mots-clés : ordonnancement, faute, checkpoint, prédiction, réplication. 


\section{Introduction}

In this report, we present scheduling algorithms to cope with faults on largescale parallel platforms. We study checkpointing and show how to derive the optimal checkpointing period. Then we explain how to combine checkpointing with fault prediction, and discuss how the optimal period is modified when this combination is used. And finally we follow the very same approach for the combination of checkpointing with replication. But wait. First, we have to help Alice out, she is having trouble with her laptop while writing her thesis.

\section{Checkpointing on a single processor}

\subsection{Alice needs help}

The most natural fault-tolerance technique when considering a fault-prone environment is to save your work periodically. This is what we (should) do in every-day's life. Alice is doing a very long and fastidious work: she is writing her $\mathrm{PhD}$ thesis, using an unreliable resource, namely a four-year-old laptop. Because she is afraid of losing her precious work if the laptop crashes, she regularly saves her work on an external disk.

At first, because she knew that her laptop could not be trusted, Alice decided to save her work on the external disk every three hours. Writing her file to disk takes approximatively three minutes. On the mid-afternoon of day 3, Alice's laptop crashed, she had to reboot it, and as a consequence she lost the last hour and a half of her work! Piqued, she decided that from now on, she would save her work on the external disk more frequently, every half hour of work instead of every three hours. But after three additional days of work without further problem, she compared what she did during the three first days, and during the three next days. She noticed that she did less work on days 4,5 and 6 than on days 1,2 and 3 (even though she lost ninety minutes of work on the third day). Alice is puzzled now: what is the best frequency to save her work?

The technique of saving intermediate work is called checkpointing. Because Alice works for a constant amount of time between two checkpoints, her technique is called periodic checkpointing. In the following, we explain why she did more work during the three first days, and how she could find the best period between each checkpoint.

\subsection{Modeling the occurrence of faults}

Computing environments, such as Alice's laptop, are prone to faults. The first question is to quantify the rate or frequency at which these faults strike. To that purpose, one uses probability distributions, and more specifically, Exponential probability distributions. The definition of $\operatorname{Exp}(\lambda)$, the Exponential distribution law of parameter $\lambda$, goes as follows:

- The probability density function is $f(t)=\lambda e^{-\lambda t} d t$ for $t \geq 0$;

$\mathrm{RR} \mathrm{n}^{\circ} 8971$ 
- The cumulative distribution function is $F(t)=1-e^{-\lambda t}$ for $t \geq 0$;

- The mean is $\mu=\frac{1}{\lambda}$.

Consider a process executing in a fault-prone environment. The time-steps at which fault strike are non-deterministic, meaning that they vary from one execution to another. To model this, we use IID (Independent and Identically Distributed) random variables $X_{1}, X_{2}, X_{3}, \ldots$. Here $X_{1}$ is the delay until the first fault, $X_{2}$ is the delay between the first and second fault, $X_{3}$ is the delay between the second and third fault, and so on. All these random variables obey the same probability distribution $\operatorname{Exp}(\lambda)$. We write $X_{i} \sim \operatorname{Exp}(\lambda)$ to express that $X_{i}$ obeys an Exponential distribution $\operatorname{Exp}(\lambda)$.

In particular, each $X_{i}$ has the same mean $\mathbb{E}\left(X_{i}\right)=\mu$. This amounts to say that, in average, a fault will strike every $\mu$ seconds. This is why $\mu$ is called the MTBF of the process, where MTBF stands for Mean Time Between Faults: one can show (see Appendix 2 for a proof) that the expected number of faults $N_{\text {faults }}(T)$ that will strike during $T$ seconds is such that

$$
\lim _{T \rightarrow \infty} \frac{N_{\text {faults }}(T)}{T}=\frac{1}{\mu}
$$

Why are Exponential distribution laws so important? This is because of their memoryless property, which writes: if $X \sim \operatorname{Exp}(\lambda)$, then $\mathbb{P}(X \geq t+s \mid X \geq s)=$ $\mathbb{P}(X \geq t)$ for all $t, s \geq 0$. This equation means that at any instant, the delay until the next fault does not depend upon the time that has elapsed since the last fault. The memoryless property is equivalent to saying that the fault rate is constant. The fault rate at time $t, \operatorname{RATE}(t)$, is defined as the (instantaneous) rate of fault for the survivors to time $t$, during the next instant of time:

$$
\operatorname{RATE}(t)=\lim _{\Delta \rightarrow 0} \frac{F(t+\Delta)-F(t)}{\Delta} \times \frac{1}{1-F(t)}=\frac{f(t)}{1-F(t)}=\lambda=\frac{1}{\mu}
$$

The fault rate is sometimes called a conditional fault rate since the denominator $1-F(t)$ is the probability that no fault has occurred until time $t$, hence converts the expression into a conditional rate, given survival past time $t$.

We have discussed Exponential laws above, but other probability laws could be used. For instance, it may not be realistic to assume that the fault rate is constant: indeed, computers, like washing machines, suffer from a phenomenon called infant mortality: the probability of fault is higher in the first weeks than later on. In other words, the fault rate is not constant but instead decreasing with time. Well, this is true up to a certain point, where another phenomenon called aging takes over: your computer, like your car, becomes more and more subject to faults after a certain amount of time: then the fault rate increases! However, after a few weeks of service and before aging, there are a few years during which it is a good approximation to consider that the fault rate is constant, and therefore to use an Exponential law $\operatorname{Exp}(\lambda)$ to model the occurrence of faults. The key parameter is the MTBF $\mu=\frac{1}{\lambda}$. 


\subsection{Problem statement}

We start by stating the problem formally. Let TIME base be the base time of the work that needs to be done, without any overhead (neither checkpoints nor faults). Assume that Alice's computer is subject to faults with a mean time between faults (MTBF) equal to $\mu$.

The time to take a checkpoint is $C$ seconds ( $C=180$ in the example). We say that the period is $T$ seconds when a checkpoint is taken each time Alice has completed $T-C$ seconds of work. When a fault occurs, the time between the last checkpoint and the fault is lost. After the fault, there is a downtime of $D$ seconds to account for the temporary unavailability (for example Alice's laptop is restarted, or the mouse is changed, or she now needs to use her brother Bob's laptop). Finally, in order to be able to resume the work, the content of the last checkpoint needs to be recovered which takes a time of $R$ seconds (the external disk is connected and the checkpoint file is read). The sum of the time lost after the fault, of the downtime and of the recovery time is denoted $T_{\text {lost }}$. All these notations are shown in Figure 1.

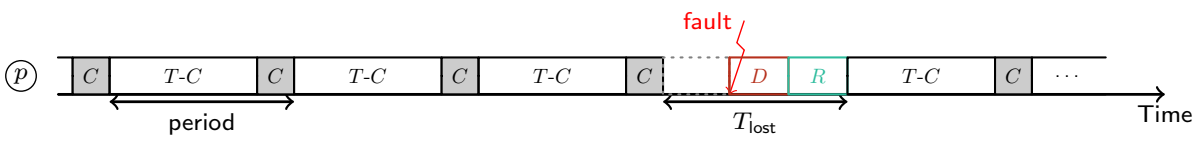

Figure 1: An execution.

\subsection{Example}

The difficulty of the problem is to trade-off between the time spent checkpointing, and the time lost in case of a fault. Consider an application such that TIME $_{\text {base }}=30$ minutes, and assume a checkpoint time of $C=3$ minutes, a downtime of $D=1$ minute and a recovery time of $R=3$ minutes.

We consider the following combinations:

$\mathrm{RR} \mathrm{n}^{\circ} 8971$ 


\section{Strategies}

1. Only one checkpoint at the end of the execution;

2. Three checkpoints during the execution, after every 10 minutes of work;

3. Five checkpoints during the execution, after every 6 minutes of work.

\section{Scenarios}

(a) A large time between faults (in this example, no fault during the execution);

(b) A medium time between faults (only one fault at the 19th minute);

(c) A small time between faults (one fault at the 19th, 42th , and 62 th minutes.).

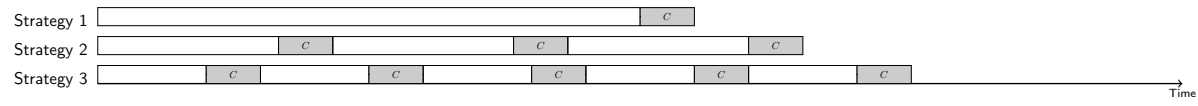

(a) Large MTBF: there are no or very few faults. Checkpointing is too expensive. The first strategy wins.

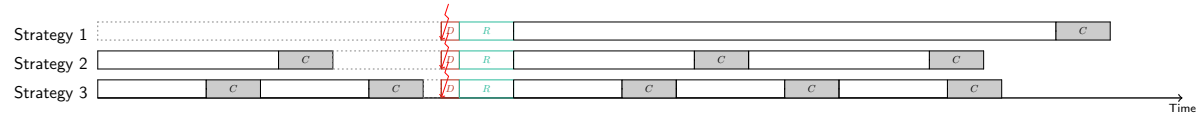

(b) Medium MTBF: there are more faults. It is good to checkpoint, but not too frequently, because of the corresponding overhead. The second strategy wins.

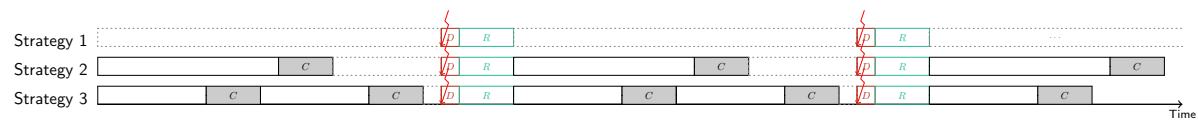

(c) Small MTBF: there are many faults. The cost of the checkpoints is paid off because the time lost due to faults is dramatically reduced. The third strategy wins.

Figure 2: The three strategies obtain different results depending upon the MTBF.

In Figure 2, we picture the execution of the application for the three different strategies, under the three different scenarios. This example shows that the lower the time between faults, the higher the frequency at which checkpoints should be taken. However, the checkpointing strategy with the smallest period is not always the best one: sometimes, there are not enough faults to pay off the overhead of frequent checkpoints.

\subsection{Solution}

Let $\operatorname{Time}_{\text {final }}(T)$ be the expectation of the total execution time of an applica-

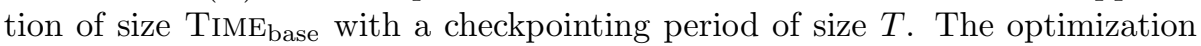


problem is to find the period $T$ minimizing $\operatorname{TIME}_{\text {final }}(T)$. However, for the sake of convenience, we rather aim at minimizing

$$
\operatorname{WAste}(T)=\frac{\operatorname{TimE}_{\text {final }}(T)-\operatorname{TimE}_{\text {base }}}{\operatorname{TimE}_{\text {final }}(T)} .
$$

This objective is called the waste as it corresponds to the fraction of the execution time that does not contribute to the progress of the application (the time wasted). Of course minimizing the waste WASTE is equivalent to minimizing the total time TIME $\mathrm{final}_{\text {, }}$, because we have

$$
\left(1-\operatorname{Waste}_{(T)}\right) \operatorname{TimE}_{\text {final }}(T)=\operatorname{TimE}_{\text {base }},
$$

but using the waste is more convenient. The waste varies between 0 and 1 . When the waste is close to 0 , it means that $\operatorname{TIME}_{\text {final }}(T)$ is very close to TIME base (which is good), whereas, if the waste is close to 1 , it means that $\operatorname{TIME}_{\text {final }}(T)$ is very large compared to TIME $E_{\text {base }}$ (which is bad).

First source of waste. Consider a fault-free execution of the application with periodic checkpointing. By definition, during each period of length $T$ we take a checkpoint, which lasts for $C$ time units, and only $T-C$ units of work are executed. Let TIMEFF be the execution time of the application in this setting. The fault-free execution time TIMEFF is equal to the time needed to execute the whole application, TimE

$$
\operatorname{TIME}_{\mathrm{FF}}=\mathrm{TIME}_{\text {base }}+N_{\mathrm{ckpt}} C,
$$

where $N_{\text {ckpt }}$ is the number of checkpoints taken. Additionally, we have

$$
N_{\mathrm{ckpt}}=\left\lceil\frac{\text { TIME }_{\mathrm{base}}}{T-C}\right\rceil \approx \frac{\text { TIME }_{\text {base }}}{T-C} .
$$

To discard the ceiling function, we assume that the execution time TIME base is large with respect to the period or, equivalently, that there are many periods during the execution. Plugging back the (approximated) value $N_{\mathrm{ckpt}}=\frac{\text { Time }_{\text {aase }}}{T-C}$, we derive that

$$
\operatorname{TiMEFF}_{F}=\frac{T}{T-C} \text { TIME }_{\text {base }}
$$

Similarly to the WASTE objective, the waste due to checkpointing in a faultfree execution, WASTEFF, is defined as the fraction of the fault-free execution time that does not contribute to the progress of the application:

$$
\text { WASTEFF }_{F}=\frac{\text { TIMEFF }_{F}-\text { TIME }_{\text {base }}}{\text { TIME }_{F F}} \Leftrightarrow\left(1-\text { WASTEFF }_{F}\right) \text { TIMEFF }_{F}=\text { TIME }_{\text {base }} .
$$

Combining Equations (2) and (3), we get:

$$
\mathrm{WASTE}_{\mathrm{FF}}=\frac{C}{T} .
$$

$\mathrm{RR} \mathrm{n}^{\circ} 8971$ 
This result is quite intuitive: every $T$ seconds, we waste $C$ for checkpointing. This calls for a very large period in a fault-free execution (even an infinite period, meaning no checkpoint at all). However, a large period also implies that a large amount of work is lost whenever a fault strikes, as we discuss now.

Second source of waste. Consider the entire execution (with faults) of the application. Let TIMEfinal denote the expected execution time of the application in the presence of faults. This execution time can be divided into two parts: (i) the execution of chunks of work of size $T-C$ followed by their checkpoint; and (ii) the time lost due to the faults. This decomposition is illustrated in Figure 3. The first part of the execution time is equal to TIMEFF. Let $N_{\text {faults }}$ be the number of faults occurring during the execution, and let $T_{\text {lost }}$ be the average time lost per fault. Then,

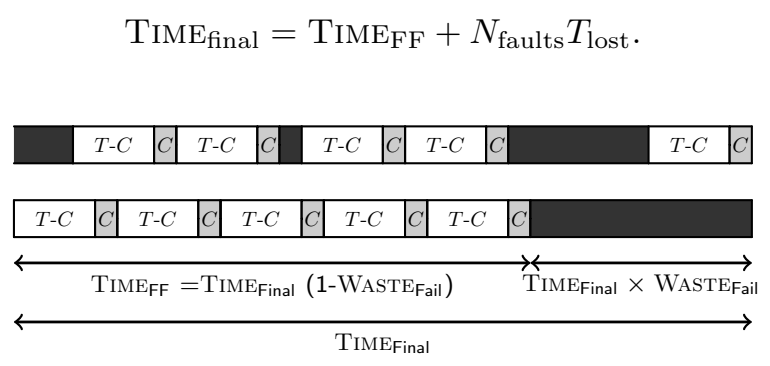

Figure 3: An execution (top), and its re-ordering (bottom), to illustrate both sources of waste. Blackened intervals correspond to time lost due to faults: downtimes, recoveries, and re-execution of work that has been lost.

In average, during a time TIME final,$N_{\text {faults }}=\frac{\text { TIMEfinal }_{\mu}}{\mu}$ faults happen (recall Equation (1)). We need to estimate $T_{\text {lost }}$ (see Figure 1). A natural estimation for the moment when the fault strikes in the period is $\frac{T}{2}$. Intuitively, faults strike anywhere in the period, hence in average they strike in the middle of the period. The proof of this result for Exponential distribution laws can be found in [8]. We conclude that $T_{\text {lost }}=\frac{T}{2}+D+R$, because after each fault there is a downtime and a recovery. This leads to:

$$
\operatorname{TIME}_{\text {final }}=\operatorname{TIME}_{\mathrm{FF}}+\frac{\text { TIMEfinal }_{\text {f }}}{\mu}\left(D+R+\frac{T}{2}\right) .
$$

Let WASTE fault be the fraction of the total execution time that is lost because of faults:

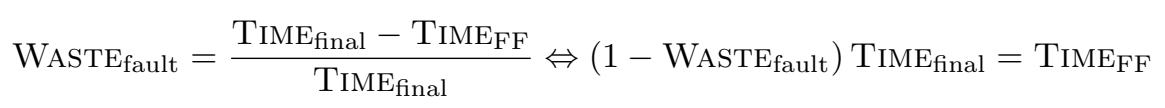

We derive:

$$
\mathrm{WASTE}_{\mathrm{fault}}=\frac{1}{\mu}\left(D+R+\frac{T}{2}\right)
$$


Equations (4) and (5) show that each source of waste calls for a different period: a large period for $\mathrm{WASTE}_{\mathrm{FF}}$, as already discussed, but a small period for WASTE $\mathrm{fault}_{\text {, }}$, to decrease the amount of work to re-execute after each fault. Clearly, a trade-off is to be found. Here is how. By definition we have

$$
\begin{aligned}
\text { WASTE } & =1-\frac{\text { TIME }_{\text {base }}}{\text { TIME }_{\text {final }}} \\
& =1-\frac{\text { TIME }_{\text {base }}}{\text { TIME }_{F F}} \frac{\text { TIMEFF }_{\text {FIME }}}{\text { TInal }} \\
& =1-\left(1-\text { WASTE }_{F F}\right)\left(1-\text { WASTE }_{\text {fault }}\right) .
\end{aligned}
$$

Altogether, we derive the final result:

$$
\begin{aligned}
\text { WASTE } & =\text { WASTEFF }_{F}+\text { WASTE }_{\text {fault }}-\text { WASTE }_{\mathrm{FF}} \text { WASTE }_{\text {fault }} \\
& =\frac{C}{T}+\left(1-\frac{C}{T}\right) \frac{1}{\mu}\left(D+R+\frac{T}{2}\right) .
\end{aligned}
$$

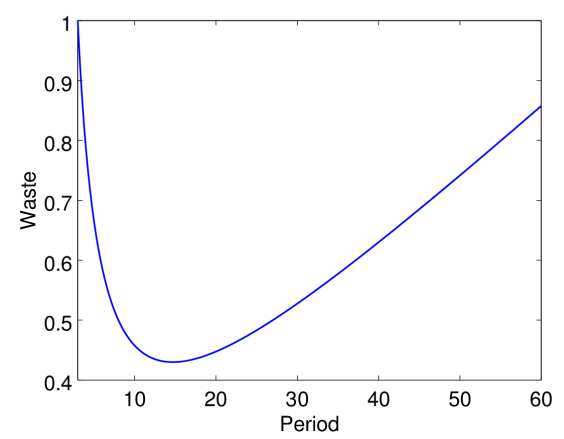

Figure 4: Waste as a function of the period $T$, for $C=3, D=1, R=3$ and $\mu=40 . T_{\mathrm{FO}} \approx 14.7$. Shorter periods increase WASTE $\mathrm{FF}_{\mathrm{F}}$ too much. Longer periods increase WASTE fault too much. $T_{\mathrm{FO}}$ achieves the best trade-off between both sources of waste.

In Figure 4, we plot WASTE as a function of the period $T$ for a set of parameters. We obtain WASTE $=\frac{u}{T}+v+w T$, where $u=C\left(1-\frac{D+R}{\mu}\right), v=$ $\frac{D+R-C / 2}{\mu}$, and $w=\frac{1}{2 \mu}$. It is easy to see that WASTE is minimized for $T=\sqrt{\frac{u}{w}}$. The First-Order (FO) formula for the optimal period is thus:

$$
T_{\mathrm{FO}}=\sqrt{2(\mu-(D+R)) C} .
$$

and the optimal waste is $\mathrm{WASTE}_{\mathrm{FO}}=2 \sqrt{u w}+v$, therefore

$$
\mathrm{WASTE}_{\mathrm{FO}}=\sqrt{\frac{2 C}{\mu}\left(1-\frac{D+R}{\mu}\right)}+\frac{D+R-C / 2}{\mu} .
$$

$\mathrm{RR} \mathrm{n}^{\circ} 8971$ 
Finally, we show in Appendix 1 why the computation above is a first order approximation.

In 1974, Young [21] obtained a different formula, namely $T_{\mathrm{FO}}=\sqrt{2 \mu C}+$ $C$. Thirty years later, Daly [8] refined Young's formula and obtained $T_{\mathrm{FO}}=$ $\sqrt{2(\mu+R) C}+C$. Equation (8) is yet another variant of the formula, which we have obtained through the computation of the waste. There is no mystery, though. None of the three formulas is correct! They represent different firstorder approximations, which collapse into the beautiful formula $T_{\mathrm{FO}}=\sqrt{2 \mu C}$ when $\mu$ is large in front of the resilience parameters $D, C$ and $R$. This latter condition is the key to the accuracy of the approximation (see Appendix 1). Let us formulate our result as a theorem:

Theorem 1. The optimal checkpointing period is $T_{\mathrm{FO}}=\sqrt{2 \mu C}+o(\sqrt{\mu})$ and the corresponding waste is $\mathrm{WASTE}_{\mathrm{FO}}=\sqrt{\frac{2 C}{\mu}}+o\left(\sqrt{\frac{1}{\mu}}\right)$.

Theorem 1 has a wide range of applications. We discuss three of them in the following sections.

\section{Checkpointing on a parallel platform}

In this section we deal with the problem of checkpointing a parallel application. We show how to reduce the optimization problem with $N$ processors to the previous problem with only one processor. Most high performance applications are tightly-coupled applications, where each processor is frequently sending messages to, and receiving messages from the other processors. This implies that the execution can progress only when all processors are up and running. This also implies that when a fault strikes one processor, the whole application must be restarted from the last checkpoint. Indeed, even though the other processors are still alive, they will very soon need some information from the faulty processor. But to catch up, the faulty processor must re-execute the work that it has lost, during which it had received messages from the other processors. But these messages are no longer available. This is why all processors have to recover from the last checkpoint and re-execute the work in parallel.

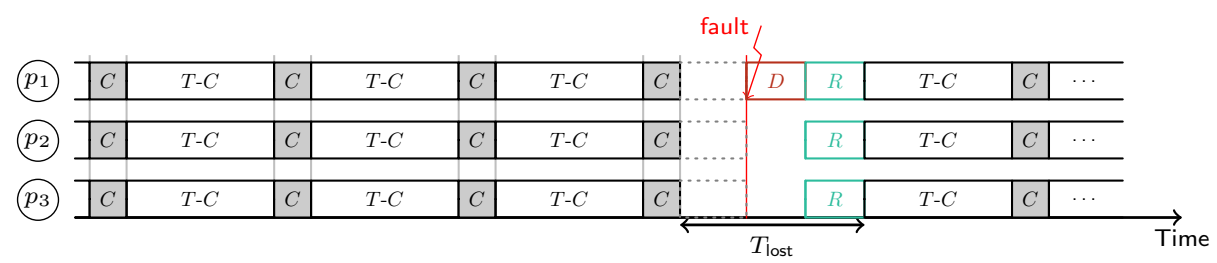

Figure 5: Behavior for a tightly coupled application.

Let us recap. Each time a fault strikes somewhere on the platform, the application stops, all processors perform a downtime and a recovery, and they 
re-execute the work during a time $T_{\text {lost }}$. This sounds familiar. We can see the whole platform as a single super-processor, very powerful (its speed is $N$ times that of individual processors) but also very prone to faults: all the faults strike this poor super-processor! See Figure 6 for an illustration.
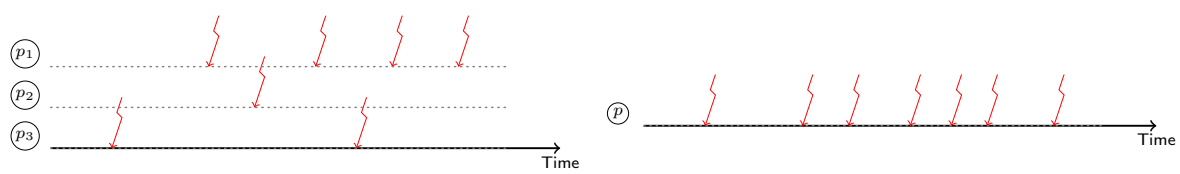

(a) Three faulty processors...

(b) ...make up for an equivalent even more faulty processor!

Figure 6: Platform model: the super-processor replaces $N=3$ processors.

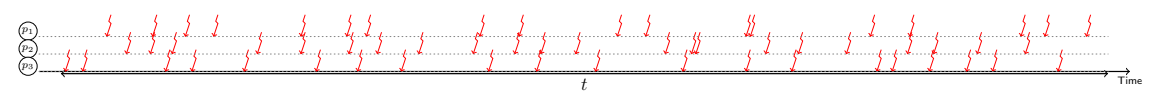

(a) If three processors have around 20 faults during a time $t\left(\mu_{\text {ind }}=\frac{t}{20}\right) \ldots$

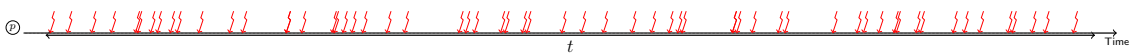

(b) ...during the same time, the equivalent processor has around 60 faults $\left(\mu=\frac{t}{60}\right)$

Figure 7: Intuition of the proof of Proposition 1.

We can apply Theorem 1 to the super-processor and determine the optimal checkpointing period as $T_{\mathrm{FO}}=\sqrt{2 \mu C}+o(\sqrt{\mu})$, where $\mu$ now is the MTBF of the super-processor. How can we compute this MTBF? Have a look at Figure 7. We see that the super-processor is hit by faults $N$ times more frequently than the individual processors. We should then conclude that its MTBF is $N$ times smaller than that of each processor. We state this result formally:

Proposition 1. Consider a platform with $N$ identical processors, each with $M T B F \mu_{\text {ind }}$. Let $\mu$ be the MTBF of the platform. Then

$$
\mu=\frac{\mu_{i n d}}{N}
$$

Proof. If the inter-arrival times of the faults on each individual processor are IID random variables (recall that IID means Independent and Identically Distributed) with distribution $\operatorname{Exp}(\lambda)$ (where $\left.\lambda=\frac{1}{\mu_{\text {ind }}}\right)$, then the inter-arrival times of the faults on the super-processor are IID random variables with distribution $\operatorname{Exp}(N \lambda)$, which will prove the result.

The arrival time of the first fault on the super-processor is a random variable $Y_{1} \sim \operatorname{Exp}(\lambda)$. This is because $Y_{1}$ is the minimum of $X_{1}^{(1)}, X_{1}^{(2)} \ldots, X_{1}^{(N)}$, where $X_{1}^{(i)}$ is the arrival time of the first fault on processor $P_{i}$. But $X_{1}^{(i)} \sim \operatorname{Exp}(\lambda)$ for all $i$, and the minimum of $N$ random variables following an Exponential distribution $\operatorname{Exp}\left(\lambda_{i}\right)$ is a random variable following an Exponential distribution $\operatorname{Exp}\left(\sum_{i=1}^{N} \lambda_{i}\right)($ see $[19$, p. 288]).

$\mathrm{RR} \mathrm{n}^{\circ} 8971$ 
The memoryless property of Exponential distributions is the key to the result for the delay between the first and second fault on the super-processor. Knowing that first fault occurred on processor $P_{1}$ at time $t$, what is the distribution of random variable for the occurrence of the first fault on processor $P_{2}$ ? The only new information if that $P_{2}$ has been alive for $t$ seconds. The memoryless property states that the distribution of the arrival time of the first fault on $P_{2}$ is not changed at all when given this information! It is still an Exponential distribution $\operatorname{Exp}(\lambda)$. Of course this holds true not only for $P_{2}$, but for each processor. And we can use the same minimum trick as for the first fault. Finally, the reasoning is the same for the third fault, and so on. This concludes the proof. We refer the reader to Appendix 3 for another proof, where we also prove Equation (1).

Proposition 1 shows that scale is the enemy of fault-tolerance. If we double up the number of components in the platform, we divide the MTBF by 2, and the minimum waste automatically increases by a factor $\sqrt{2} \approx 1.4$ (see Equation $(9)$ ). And this assumes that the checkpoint time $C$ remains constant. With twice as many processors, there is twice more data to write onto stable storage, hence the aggregated I/O bandwidth of the platform must be doubled to match this requirement.

\section{Fault prediction}

A possible way to cope with the numerous faults and their impact on the execution time is to try and predict them. In this section we do not explain how this is done, although the interested reader may find some answers in [12, 22, 24]. To give a single example, the reader may think of a sensor detecting unexpected heat and reporting that something bad is going to happen soon.

A fault predictor (or simply a predictor) is a mechanism that warns the user about upcoming faults on the platform. More specifically, a predictor is characterized by two key parameters, its recall $r$, which is the fraction of faults that are indeed predicted, and its precision $p$, which is the fraction of predictions that are correct (i.e., correspond to actual faults). In this section, we discuss how to combine checkpointing and prediction to decrease the platform waste.

We start with a few definitions. Let $\mu_{\mathrm{P}}$ be the mean time between predicted events (both true positive and false positive), and $\mu_{\mathrm{NP}}$ be the mean time between unpredicted faults (false negative). The relations between $\mu_{\mathrm{P}}, \mu_{\mathrm{NP}}, \mu, r$ and $p$ are as follows:

- Rate of unpredicted faults: $\frac{1}{\mu_{\mathrm{NP}}}=\frac{1-r}{\mu}$, since $1-r$ is the fraction of faults that are unpredicted;

- Rate of predicted faults: $\frac{r}{\mu}=\frac{p}{\mu_{\mathrm{P}}}$, since $r$ is the fraction of faults that are predicted, and $p$ is the fraction of fault predictions that are correct.

To illustrate all these definitions, consider the time interval below and the different events occurring: 


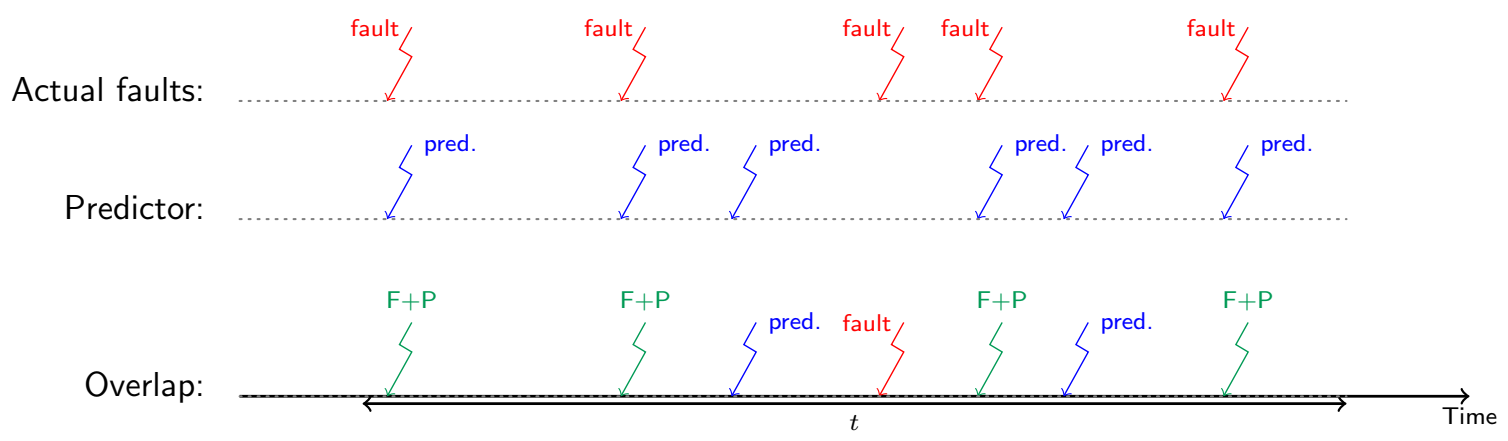

During this time interval of length $t$, the predictor predicts six faults, and there were five actual faults. One fault was not predicted. This gives approximatively: $\mu=\frac{t}{5}, \mu_{\mathrm{P}}=\frac{t}{6}$, and $\mu_{\mathrm{NP}}=t$. For this predictor, the recall is $r=\frac{4}{5}$ (green arrows over red arrows), and its precision is $p=\frac{4}{6}$ (green arrows over blue arrows).

Now, given a fault predictor of parameters $p$ and $r$, can we improve the waste? More specifically, how to modify the periodic checkpointing algorithm to get better results? In order to answer these questions, we introduce proactive checkpointing: when there is a prediction, we assume that the prediction is given early enough so that we have time for a checkpoint of size $C_{p}$ (which can be different from $C$ ). We consider the following simple algorithm:

- While no fault prediction is available, checkpoints are taken periodically with period $T$;

- When a fault is predicted, we take a proactive checkpoint (of length $C_{p}$ ) as late as possible, so that it completes right at the time when the fault is predicted to strike. After this checkpoint, we complete the execution of the period (see Figures $8 \mathrm{~b}$ and $8 \mathrm{c}$ );

We compute the expected waste as before. We reproduce Equation (6) below:

$$
\text { WASTE }=\text { WASTEFF }_{F}+\text { WASTE }_{\text {fault }}-\text { WASTEFF }_{\text {FASTE fault }}
$$

While the value of WASTEFF is unchanged $\left(\right.$ WASTEFF $_{F}=\frac{C}{T}$ ), the value of WASTE $_{\text {fault }}$ is modified because of predictions. As illustrated in Figure 8, there are different scenarios that contribute to WASTEfault. We classify them as follows:

(1) Unpredicted faults: This overhead occurs each time an unpredicted fault strikes, that is, on average, once every $\mu_{\mathrm{NP}}$ seconds. Just as in Equation (5), the corresponding waste is $\frac{1}{\mu_{\mathrm{NP}}}\left[\frac{T}{2}+D+R\right]$.

(2) Predictions: We now compute the overhead due to a prediction. If the prediction is an actual fault (with probability $p$ ), we lose $C_{p}+D+R$

$\mathrm{RR} \mathrm{n}^{\circ} 8971$ 


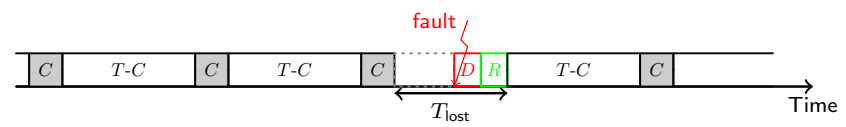

(a) Unpredicted fault

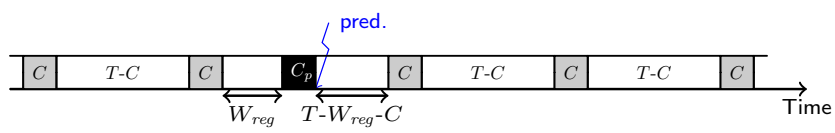

(b) Prediction taken into account - no actual fault

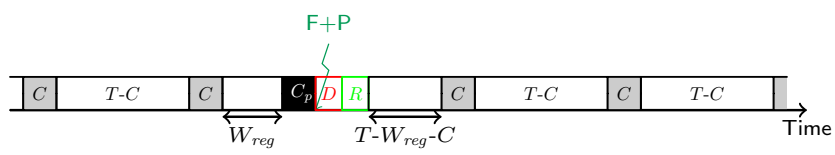

(c) Prediction taken into account - with actual fault

Figure 8: Actions taken for the different event types.

seconds, but if it is not (with probability $1-p$ ), we lose the unnecessary extra checkpoint time $C_{p}$. Hence

$$
T_{\text {lost }}=p\left(C_{p}+D+R\right)+(1-p) C_{p}=C_{p}+p(D+R)
$$

We derive the final value of WASTE$_{\text {fault }}$ :

$$
\begin{aligned}
\text { WASTE }_{\mathrm{fault}} & =\frac{1}{\mu_{\mathrm{NP}}}\left(\frac{T}{2}+D+R\right)+\frac{1}{\mu_{\mathrm{P}}}\left(C_{p}+p(D+R)\right) \\
& =\frac{1-r}{\mu}\left(\frac{T}{2}+D+R\right)+\frac{r}{p \mu}\left(C_{p}+p(D+R)\right) \\
& =\frac{1}{\mu}\left((1-r) \frac{T}{2}+D+R+\frac{r C_{p}}{p}\right)
\end{aligned}
$$

We can now plug this expression back into Equation (11):

$$
\begin{aligned}
\text { WASTE } & =\text { WASTE }_{\mathrm{FF}}+\text { WASTE }_{\mathrm{fault}}-\text { WASTE }_{\mathrm{FF}} \text { WASTE }_{\mathrm{fault}} \\
& =\frac{C}{T}+\left(1-\frac{C}{T}\right) \frac{1}{\mu}\left(D+R+\frac{r C_{p}}{p}+\frac{(1-r) T}{2}\right) .
\end{aligned}
$$

To compute the value of $T_{\mathrm{FO}}^{p}$, the period that minimizes the total waste, we use the same reasoning as in Section 2.5 and obtain:

$$
T_{\mathrm{FO}}^{p}=\sqrt{\frac{2\left(\mu-\left(D+R+\frac{r C_{p}}{p}\right)\right) C}{1-r}} .
$$

We observe the similarity of this result with the value of $T_{\mathrm{FO}}$ from Equation (8). If $\mu$ is large in front of the resilience parameters, we derive that $T_{\mathrm{FO}}^{p}=\sqrt{\frac{2 \mu C}{1-r}}$. 
This tells us that the recall is more important than the precision. If the predictor is capable of predicting, say, $84 \%$ of the faults, then $r=0.84$ and $\sqrt{1-r}=0.4$. The optimal period is increased by $40 \%$, and the waste is decreased by the same factor. Prediction can help! See Appendix 4 for further information.

\section{Replication}

Another possible way to cope with the numerous faults and their impact on the execution time is to use replication. Replication consists in duplicating all computations. Processors are grouped by pairs, such as each processor has a buddy (another processor performing exactly the same computations, receiving the same messages, etc). See Figure 9 for an illustration. We say that the two processes in a given pair are replicas. When a processor is hit by a fault, its buddy is not impacted. The execution of the application can still progress, until the buddy itself is hit by a fault later on. This sounds quite expensive: by definition, half of the resources are wasted (and this does not include the overhead of maintaining a consistent state between the two processors of each pair). At first sight, the idea of using replication on a large parallel platform is puzzling: who is ready to waste half of these expensive supercomputers?

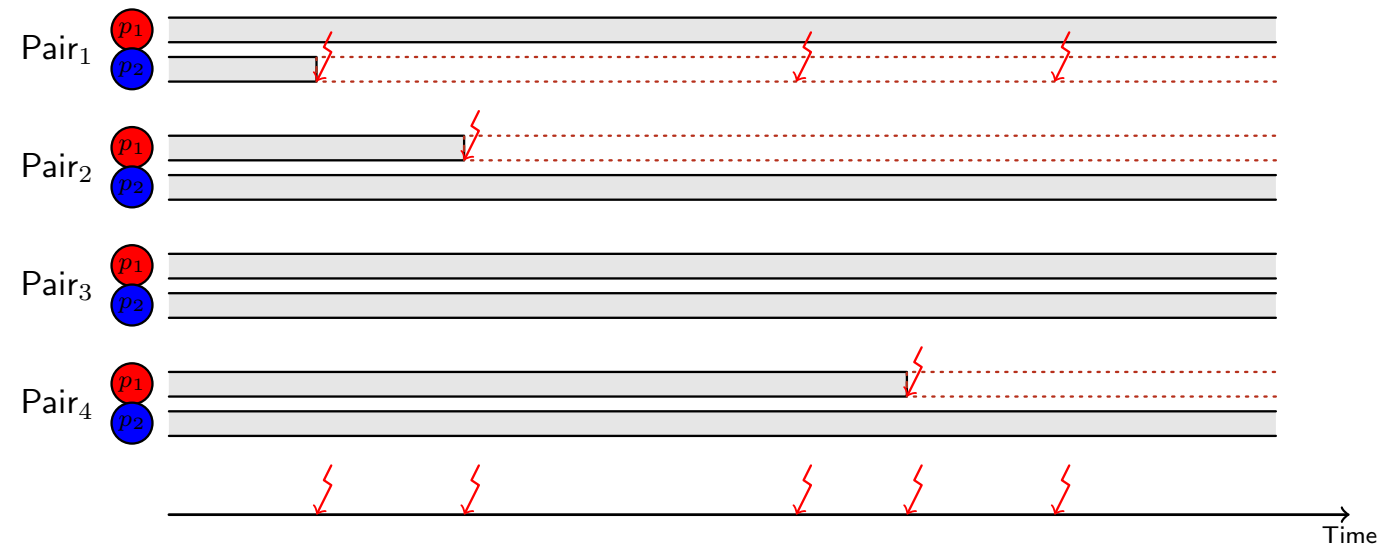

Figure 9: Processor pairs for replication: each blue processor is paired with a red processor. In each pair, both processors do the same work.

In this section, we explain how replication can be used in conjunction with checkpointing and under which conditions it becomes profitable. In order to do this, we compare the checkpointing technique introduced earlier to the replication technique.

A perfectly parallel application is an application such that in a failure-free, checkpoint-free environment, the time to execute the application (TIME ${ }_{\text {Base }}$ )

$\mathrm{RR} \mathrm{n}^{\circ} 8971$ 
decreases linearly with the number of processors. More precisely:

$$
\operatorname{TimE}_{\text {base }}(N)=\frac{\operatorname{TimE}_{\text {base }}(1)}{N} \text {. }
$$

Consider the execution of a perfectly parallel application on a platform with $N=$ $2 n$ processors, each with individual MTBF $\mu_{\text {ind }}$. As in the previous sections, the

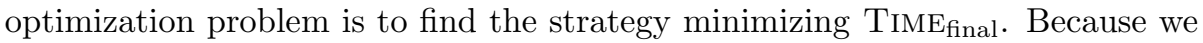
compare two approaches using a different number of processors, we introduce the Throughput, which is defined as the total number of useful flops per second:

$$
\text { Throughput }=\frac{\operatorname{TimE}_{\text {base }}(1)}{\text { TimE }_{f i n a l}}
$$

Note that for an application executing on $N$ processors, Throughput $=N(1-$ WASTE $)$.

The standard approach, as seen before, is to use all $2 n$ processors to fully parallelize the execution of the application on the platform. This would be optimal in a fault-free environment, but we are required to checkpoint frequently because faults repeatedly strike the $N$ processors. According to Proposition 1, the platform MTBF is $\mu=\frac{\mu_{\text {ind }}}{N}$. According to Theorem 1, the waste is (approximately) WASTE $=\sqrt{\frac{2 C}{\mu}}=\sqrt{\frac{2 C N}{\mu_{\text {ind }}}}$. We have:

$$
\text { ThroughPUT }_{\text {Std }}=N\left(1-\sqrt{\frac{2 C N}{\mu_{\text {ind }}}}\right)
$$

The second approach uses replication. There are $n$ pairs of processors, all computations are executed twice, hence only half the processors produce useful flops. One way to see the replication technique is as if there were half the processors using only the checkpoint technique, with a different (potentially higher) mean time between faults, $\mu_{\text {rep }}$. Hence, the throughput ThroughPUT Rep $_{\text {of }}$ this approach writes:

$$
\text { ThroUGHPUT }_{\text {Rep }}=\frac{N}{2}\left(1-\sqrt{\frac{2 C}{\mu_{\text {rep }}}}\right)
$$

In fact, rather than MTBF, we should say MTTI, for Mean Time To Interruption. As already mentioned, a single fault on the platform does not interrupt the application, because the replica of the faulty processor is still alive. What is the value of MNFTI, the Mean Number of Faults To Interruption, i.e., the mean number of faults that should strike the platform until there is a replica pair whose processors have both been hit? If we find how to compute MNFTI, we are done, because we know that

$$
\mu_{\mathrm{rep}}=M N F T I \times \mu=M N F T I \times \frac{\mu_{\text {ind }}}{N}
$$

We make an analogy with a balls-into-bins problem to compute MNFTI. The classical problem is the following: what is the expected number of balls 


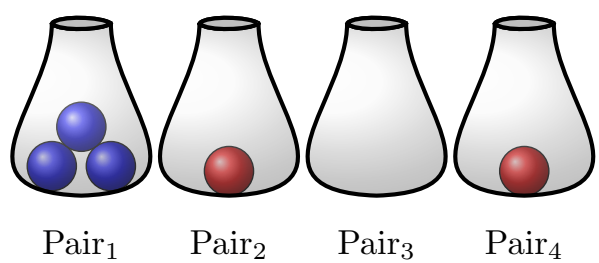

Figure 10: Modeling the state of the platform of Figure 9 as a balls-into-bins problem. We put a red ball in bin $\operatorname{Pair}_{i}$ when there is a fault on its red processor $p_{1}$, and a blue ball when there is a fault on its blue processor $p_{2}$. As long as no bin has received a ball of each color, the game is on.

that you will need, if you throw these balls randomly into $n$ bins, until one bins gets two balls? The answer to this question is given by Ramanujan's QFunction [11], and is equal to $\lceil q(n)\rceil$ where $q(n)=\frac{2}{3}+\sqrt{\frac{\pi n}{2}}+\sqrt{\frac{\pi}{288 n}}-\frac{4}{135 n}+\ldots$. When $n=365$, this is the birthday problem where balls are persons and bins are calendar dates; in the best case, one needs two persons; in the worst case, one needs $n+1=366$ persons; on average, one needs $\lceil q(n)\rceil=25$ persons. $^{1}$

In the replication problem, the bins are the processor pairs, and the balls are the faults. However, the analogy stops here. The problem is more complicated, see Figure 10 to see why. Each processor pair is composed of a blue processor and of a red processor. Faults are (randomly) colored blue or red too. When a fault strikes a processor pair, we need to know which processor inside that pair: we decide that it is the one of the same color as the fault. Blue faults strike blue processors, and red faults strike red processors. We now understand that we may need more than two faults hitting the same pair to interrupt the application: we need one fault of each color. The balls-and-bins problem to compute MNFTI is the following: what is the expected number of red and blue balls that you will need, if you throw these balls randomly into $n$ bins, until one bins gets one red ball and one blue ball? To the best of our knowledge, there is no closed-form solution to answer this question, but a recursive computation does the job:

Proposition 2. $M N F T I=\mathbb{E}(N F T I \mid 0)$ where

$$
\mathbb{E}\left(N F T I \mid n_{f}\right)= \begin{cases}2 & \text { if } n_{f}=n, \\ \frac{2 n}{2 n-n_{f}}+\frac{2 n-2 n_{f}}{2 n-n_{f}} \mathbb{E}\left(N F T I \mid n_{f}+1\right) & \text { otherwise. }\end{cases}
$$

Proof. Let $\mathbb{E}\left(N F T I \mid n_{f}\right)$ be the expectation of the number of faults needed to interrupt the application, knowing that the application is still running and that faults have already hit $n_{f}$ different processor pairs. Because each pair initially has 2 replicas, this means that $n_{f}$ different pairs are no longer replicated, and

\footnotetext{
${ }^{1}$ As a side note, one needs only 23 persons for the probability of a common birthday to reach 0.5 (a question often asked during geek evenings).
} 
that $n-n_{f}$ are still replicated. Overall, there are $n_{f}+2\left(n-n_{f}\right)=2 n-n_{f}$ processors still running.

The case $n_{f}=n$ is simple. In this case, all pairs have already been hit, and all pairs have only one of their two initial replicas still running. A new fault will hit such a pair. Two cases are then possible:

1. The fault hits the running processor. This leads to an application interruption, and in this case $\mathbb{E}(N F T I \mid n)=1$.

2. The fault hits the processor that has already been hit. Then the fault has no impact on the application. The MNFTI of this case is then: $\mathbb{E}(N F T I \mid n)=1+\mathbb{E}(N F T I \mid n)$.

The probability of fault is uniformly distributed between the two replicas, and thus between these two cases. Weighting the values by their probabilities of occurrence yields:

$$
\mathbb{E}(N F T I \mid n)=\frac{1}{2} \times 1+\frac{1}{2} \times(1+\mathbb{E}(N F T I \mid n)),
$$

hence $\mathbb{E}(N F T I \mid n)=2$.

For the general case $0 \leq n_{f} \leq n-1$, either the next fault hits a new pair, i.e., a pair whose 2 processors are still running, or it hits a pair that has already been hit, hence with a single processor running. The latter case leads to the same sub-cases as the $n_{f}=n$ case studied above. The fault probability is uniformly distributed among the $2 n$ processors, including the ones already hit. Hence the probability that the next fault hits a new pair is $\frac{2 n-2 n_{f}}{2 n}$. In this case, the expected number of faults needed to interrupt the application fail is one (the considered fault $)$ plus $\mathbb{E}\left(N F T I \mid n_{f}+1\right)$. Altogether we have:

$\mathbb{E}\left(N F T I \mid n_{f}\right)=\frac{2 n-2 n_{f}}{2 n} \times\left(1+\mathbb{E}\left(N F T I \mid n_{f}+1\right)\right)+\frac{2 n_{f}}{2 n} \times\left(\frac{1}{2} \times 1+\frac{1}{2}\left(1+\mathbb{E}\left(N F T I \mid n_{f}\right)\right)\right)$.

Therefore,

$$
\mathbb{E}\left(N F T I \mid n_{f}\right)=\frac{2 n}{2 n-n_{f}}+\frac{2 n-2 n_{f}}{2 n-n_{f}} \mathbb{E}\left(N F T I \mid n_{f}+1\right) .
$$

Let us compare the throughput of each approach with an example. From Equations (12) and (13), we have

THROUGHPUT Rep $_{\text {RHROUGHPUT }} \geq$ Thtd $\Leftrightarrow\left(1-\sqrt{\frac{2 C N}{M N F T I \mu_{\text {ind }}}}\right) \geq 2\left(1-\sqrt{\frac{2 C N}{\mu_{\text {ind }}}}\right)$

which we rewrite into

$$
C \geq \frac{\mu_{\text {ind }}}{2 N} \frac{1}{\left(2-\frac{1}{\sqrt{M N F T I}}\right)^{2}}
$$

Take a parallel machine with $N=2^{20}$ processors. This is a little more than one million processors, but this corresponds to the size of the largest platforms 
today. Using Proposition 2, we compute MNFTI $=1284.4$ Assume that the individual MTBF is 10 years, or in seconds $\mu_{\text {ind }}=10 \times 365 \times 24 \times 3600$. After some painful computations, we derive that replication is more efficient if the checkpoint time is greater than 293 seconds (around 6 minutes). This sets a target both for architects and checkpoint protocol designers.

Maybe you can say that $\mu_{\text {ind }}=10$ years is pessimistic, because we rather observe that $\mu_{\text {ind }}=100$ years in current supercomputers. Because $\mu_{\text {ind }}=100$ years allows to checkpoint up to one hour, you would decide that replication is not worth it. But maybe you can also say that $\mu_{\text {ind }}=10$ years is optimistic for processors equipped with thousands of cores and rather take $\mu_{\text {ind }}=1$ year. In that case, unless you checkpoint in less than 30 seconds, better be prepared for replication. The beauty of performance models is that you can decide which approach is better without bias nor a-priori, simply by plugging your own parameters into Equation (14).

\section{Conclusion}

In this report, we have dealt with fail-stop faults, i.e. faults that cause the application to crash and require to repair the resource or to find a spare one, and to re-execute work from some state of the application that had been previously saved. Other techniques involve to reconstruct the data lost by the failing processor from redundant information (e.g., checksums) maintained by the other processors. While unrecoverable, a fail-stop error has the nice characteristic that it can be detected immediately. On the contrary, a silent error, a.k.a. silent data corruption, gets unnoticed until it manifests after some random delay, e.g. because corrupted data is activated. Silent errors come from many sources, from errors in the arithmetic unit (due to low voltages) to bit flips in cache (due to cosmic radiation). Silent errors are difficult to detect, and because of the detection latency, they are even more difficult to correct. We refer the interested reader to studies such as $[5,13]$ to know more about the fascinating problems and solution techniques in the area of fault-tolerant computing at very large scale. Exascale $\left(10^{18}\right.$ operations per second, which requires one million of processors, each with one thousand cores) is such a very large scale, but it is the scale of future-generation machines that will be with us in less than 10 years. Thus this area is extremely important, and clever scheduling techniques are needed to help solve all the problems. Alice needs more help ${ }^{2}$.

\section{Further reading}

In this section, we provide the reader who would like to know more about faulttolerance, with a few key references. A well documented paper is [3], which gives an overview of many possible approaches. To cope with fail-stop failures, saving redundant information is always needed to avoid costly re-executions

\footnotetext{
${ }^{2} \mathrm{By}$ the way, there is a nice little exercise in Appendix 6 if you are motivated to help.
} 
from scratch. This can be done (i) either at every instant, and this is replication, or even triplication, the method of choice for critical systems [18]; (ii) or at some well-chosen time-steps, and this is checkpointing. Checkpointing protocols come in many flavors, and we refer to [7] for the pioneering paper. As studied in this paper, checkpointing can be combined with prediction, or with replication.

Replication and checkpointing are general-purpose methods, meaning that they do not require any knowledge of the application. On the contrary, applicationspecific methods can use information about the application to enforce faulttolerance at a lower cost than general-purpose methods. Application-specific methods can use a refined form of general-purpose methods. For instance, one can replicate only some critical tasks (as opposed to all tasks) in the case of scientific workflows. Or one can decide to checkpoint only when the amount of data to be saved is smaller, e.g., at the end rather than in the middle of an iteration, for a sparse iterative solver. But application-specific methods can also use brand new approaches. The prominent example in HPC is ABFT, which stands for Algorithm Based Fault-Tolerance [15]: for dense linear algebra kernels such as matrix product or Cholesky/LU factorization, the idea is to use checksums of matrix row and columns. When a processor crashes, the checksums are used to reconstruct the data, and there is no re-execution at all. The beauty of ABFT is that checksums can be maintained on the fly, by applying the same transformations to them as to the original matrix. Of course this works only for linear kernels.

Finally, for the more adventurous reader, the recent monograph [16] offers a complete panorama of fault-tolerance methods for HPC. While this field is very active, much progress is needed before we can efficiently use these big machines that are (slowly but inexorably) coming into our life. Alice needs more help, remember!

\section{References}

[1] Guillaume Aupy, Yves Robert, Frédéric Vivien, and Dounia Zaidouni. Checkpointing strategies with prediction windows. In Dependable Computing (PRDC), 2013 IEEE 19th Pacific Rim International Symposium on, pages 1-10. IEEE, 2013.

[2] Guillaume Aupy, Yves Robert, Frédéric Vivien, and Dounia Zaidouni. Checkpointing algorithms and fault prediction. Journal of Parallel and Distributed Computing, 74(2):2048-2064, 2014.

[3] A. Avizienis, J. C. Laprie, B. Randell, and C. Landwehr. Basic concepts and taxonomy of dependable and secure computing. IEEE Trans. Dependable and Secure Computing, 1(1):11-33, 2004.

[4] Marin Bougeret, Henri Casanova, Mikael Rabie, Yves Robert, and Frédéric Vivien. Checkpointing strategies for parallel jobs. In Proceedings of SC'11, 2011. 
[5] Franck Cappello, Al Geist, Bill Gropp, Laxmikant V. Kalé, Bill Kramer, and Marc Snir. Toward Exascale Resilience. Int. Journal of High Performance Computing Applications, 23(4):374-388, 2009.

[6] Henri Casanova, Yves Robert, Frédéric Vivien, and Dounia Zaidouni. Combining process replication and checkpointing for resilience on exascale systems. Research report RR-7951, INRIA, May 2012.

[7] K. M. Chandy and L. Lamport. Distributed snapshots: Determining global states of distributed systems. In Transactions on Computer Systems, volume 3(1), pages 63-75. ACM, February 1985.

[8] J. T. Daly. A higher order estimate of the optimum checkpoint interval for restart dumps. FGCS, 22(3):303-312, 2004.

[9] C. Engelmann, H. H. Ong, and S. L. Scorr. The case for modular redundancy in large-scale highh performance computing systems. In Proc. of the 8th IASTED Infernational Conference on Parallel and Distributed Computing and Networks (PDCN), pages 189-194, 2009.

[10] K. Ferreira, J. Stearley, J. H. III Laros, R. Oldfield, K. Pedretti, R. Brightwell, R. Riesen, P. G. Bridges, and D. Arnold. Evaluating the Viability of Process Replication Reliability for Exascale Systems. In Proc. of the ACM/IEEE SC Conf., 2011.

[11] P. Flajolet, P. J. Grabner, P. Kirschenhofer, and H. Prodinger. On Ramanujan's Q-Function. J. Computational and Applied Mathematics, 58:103-116, 1995.

[12] A. Gainaru, F. Cappello, and W. Kramer. Taming of the shrew: Modeling the normal and faulty behavior of large-scale hpc systems. In Proc. IPDPS'12, 2012.

[13] Ana Gainaru, Franck Cappello, Marc Snir, and William Kramer. Failure prediction for hpc systems and applications: Current situation and open issues. Int. J. High Perform. Comput. Appl., 27(3):273-282, 2013.

[14] F. Gärtner. Fundamentals of fault-tolerant distributed computing in asynchronous environments. ACM Computing Surveys, 31(1), 1999.

[15] Kuang-Hua Huang and J. A. Abraham. Algorithm-based fault tolerance for matrix operations. IEEE Trans. Computers, 33(6):518-528, 1984.

[16] Thomas Hérault and Yves Robert, editors. Fault-Tolerance Techniques for High-Performance Computing, Computer Communications and Networks. Springer Verlag, 2015.

[17] D. Kondo, A. Chien, and H. Casanova. Scheduling Task Parallel Applications for Rapid Application Turnaround on Enterprise Desktop Grids. J. Grid Computing, 5(4):379-405, 2007.

$\mathrm{RR}^{\circ} 8971$ 
[18] R. E. Lyons and W. Vanderkulk. The use of triple-modular redundancy to improve computer reliability. IBM J. Res. Dev., 6(2):200-209, 1962.

[19] Sheldon M. Ross. Introduction to Probability Models, Eleventh Edition. Academic Press, 2009.

[20] B. Schroeder and G. Gibson. Understanding failures in petascale computers. Journal of Physics: Conference Series, 78(1), 2007.

[21] John W. Young. A first order approximation to the optimum checkpoint interval. Comm. of the ACM, 17(9):530-531, 1974.

[22] Li Yu, Ziming Zheng, Zhiling Lan, and S. Coghlan. Practical online failure prediction for blue gene/p: Period-based vs event-driven. In Dependable Systems and Networks Workshops (DSN-W), pages 259-264, 2011.

[23] Z. Zheng and Z. Lan. Reliability-aware scalability models for high performance computing. In Proc. of the IEEE Conference on Cluster Computing, 2009.

[24] Ziming Zheng, Zhiling Lan, R. Gupta, S. Coghlan, and P. Beckman. A practical failure prediction with location and lead time for blue gene/p. In Dependable Systems and Networks Workshops (DSN-W), pages 15-22, 2010.

\section{Appendix 1: First-order approximation of $T_{\mathrm{FO}}$}

It is interesting to point out why the value of $T_{\mathrm{FO}}$ given by Equation (8) is a firstorder approximation, even for large jobs. Indeed, there are several restrictions for the approach to be valid:

- We have stated that the expected number of faults during execution is

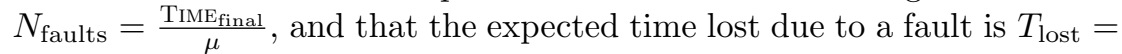
$\frac{T}{2}+D+R$. Both statements are true individually, but the expectation of a product is the product of the expectations only if the random variables are independent, which is not the case here because TIME final $_{\text {depends }}$ upon the fault inter-arrival times.

- In Equation (4), we have to enforce $C \leq T$ in order to have WASTEFF $\leq 1$.

- In Equation (5), we have to enforce $D+R \leq \mu$ in order to have WASTEfault $\leq$ 1. In addition, we must cap the period to enforce this latter constraint. Intuitively, we need $\mu$ to be large enough for Equation (5) to make sense. However, for large-scale platforms, regardless of the value of the individual MTBF $\mu_{\text {ind }}$, there is always a threshold in the number of components $N$ above which the platform MTBF, $\mu=\frac{\mu_{\text {ind }}}{N}$, becomes too small for Equation (5) to be valid.

- Equation (5) is accurate only when two or more faults do not take place within the same period. Although unlikely when $\mu$ is large in front of $T$, the possible occurrence of many faults during the same period cannot be eliminated. 
To ensure that the condition of having at most a single fault per period is met with a high probability, we cap the length of the period: we enforce the condition $T \leq \alpha \mu$, where $\alpha$ is some tuning parameter chosen as follows. The number of faults during a period of length $T$ can be modeled as a Poisson process of parameter $\beta=\frac{T}{\mu}$. The probability of having $k \geq 0$ faults is $P(X=k)=$

$\frac{\beta^{k}}{k !} e^{-\beta}$, where $X$ is the random variable showing the number of faults. Hence the probability of having two or more faults is $\pi=P(X \geq 2)=1-(P(X=$ $0)+P(X=1))=1-(1+\beta) e^{-\beta}$. If we assume $\alpha=0.27$ then $\pi \leq 0.03$, hence a valid approximation when bounding the period range accordingly. Indeed, with such a conservative value for $\alpha$, we have overlapping faults for only $3 \%$ of the checkpointing segments in average, so that the model is quite reliable. For consistency, we also enforce the same type of bound on the checkpoint time, and on the downtime and recovery: $C \leq \alpha \mu$ and $D+R \leq \alpha \mu$. However, enforcing these constraints may lead to use a sub-optimal period: it may well be the case that the optimal period $\sqrt{2(\mu-(D+R)) C}$ of Equation (8) does not belong to the admissible interval $[C, \alpha \mu]$. In that case, the waste is minimized for one of the bounds of the admissible interval. This is because, as seen from Equation (7), the waste is a convex function of the period.

We conclude this discussion on a positive note. While capping the period, and enforcing a lower bound on the MTBF, is mandatory for mathematical rigor, simulations in [2] show that actual job executions can always use the value from Equation (8), accounting for multiple faults whenever they occur by re-executing the work until success. The first-order model turns out to be surprisingly robust!

\section{Appendix 2: Optimal value of $T_{\text {FO }}$}

There is a beautiful method to compute the optimal value of $T_{\text {FO }}$ accurately. First we show how to compute the expected time $\mathbb{E}(\operatorname{Time}(T-C, C, D, R, \lambda))$ to execute a work of duration $T-C$ followed by a checkpoint of duration $C$, given the values of $C, D$, and $R$, and a fault distribution $\operatorname{Exp}(\lambda)$. If a fault interrupts a given trial before success, there is a downtime of duration $D$ followed by a recovery of length $R$. We assume that faults can strike during checkpoint and recovery, but not during downtime.

\section{Proposition 3.}

$$
\mathbb{E}(\operatorname{Time}(T-C, C, D, R, \lambda))=e^{\lambda R}\left(\frac{1}{\lambda}+D\right)\left(e^{\lambda T}-1\right) .
$$

Proof. For simplification, we write Time instead of Time $(T-C, C, D, R, \lambda)$ in the proof below. Consider the following two cases:

(i) Either there is no fault during the execution of the period, then the time needed is exactly $T$;

(ii) Or there is one fault before successfully completing the period, then some additional delays are incurred. More specifically, as seen for the first order 
approximation, there are two sources of delays: the time spent computing by the processors before the fault (accounted for by variable TIMElost), and the time spent for downtime and recovery (accounted for by variable TIME $_{\text {rec }}$ ). Once a successful recovery has been completed, there still remain $T-C$ units of work to execute.

Thus Time obeys the following recursive equation:

$$
\text { Time }= \begin{cases}T & \text { if there is no fault } \\ \text { Time }_{\text {lost }}+\text { TIME }_{\text {rec }}+\text { Time } & \text { otherwise }\end{cases}
$$

TIMElost denotes the amount of time spent by the processors before the first fault, knowing that this fault occurs within the next $T$ units of time. In other terms, it is the time that is wasted because computation and checkpoint were not successfully completed (the corresponding value in Figure 1 is $T_{\text {lost }}-D-R$ ).

TIME $_{\text {rec }}$ represents the amount of time needed by the system to recover from the fault (the corresponding value in Figure 1 is $D+R$ ).

The expectation of TIME can be computed from Equation (15) by weighting each case by its probability to occur:

$$
\begin{aligned}
\mathbb{E}(\text { Time }) & =\mathbb{P}(\text { no fault }) \cdot T+\mathbb{P}(\text { a fault strikes }) \cdot \mathbb{E}\left(\text { TIME }_{\text {lost }}+\text { TIME }_{\text {rec }}+\text { TimE }\right) \\
& =e^{-\lambda T} T+\left(1-e^{-\lambda T}\right)\left(\mathbb{E}\left(\text { TIME }_{\text {lost }}\right)+\mathbb{E}\left(\text { TIME }_{\text {rec }}\right)+\mathbb{E}(\text { TImE })\right)
\end{aligned}
$$

which simplifies into:

$$
\mathbb{E}(T)=T+\left(e^{\lambda T}-1\right)\left(E\left(\text { TIME }_{\text {lost }}\right)+E\left(\text { TIME }_{\text {rec }}\right)\right)
$$

We have $\mathbb{E}\left(\right.$ TImE $\left._{\text {lost }}\right)=\int_{0}^{\infty} x \mathbb{P}(X=x \mid X<T) d x=\frac{1}{\mathbb{P}(X<T)} \int_{0}^{T} e^{-\lambda x} d x$, and $\mathbb{P}(X<T)=1-e^{-\lambda T}$. Integrating by parts, we derive that

$$
\mathbb{E}\left(\operatorname{TIME}_{\text {lost }}\right)=\frac{1}{\lambda}-\frac{T}{e^{\lambda T}-1}
$$

Next, the reasoning to compute $\mathbb{E}\left(\mathrm{TIME}_{\mathrm{rec}}\right)$, is very similar to $\mathbb{E}$ (TIME) (note that there can be no fault during $D$ but there can be during $R$ ):

$$
\mathbb{E}\left(\mathrm{TimE}_{\mathrm{rec}}\right)=e^{-\lambda R}(D+R)+\left(1-e^{-\lambda R}\right)\left(D+\mathbb{E}\left(R_{\text {lost }}\right)+\mathbb{E}\left(\mathrm{TimE}_{\mathrm{rec}}\right)\right)
$$

Here, $R_{\text {lost }}$ is the amount of time lost to executing the recovery before a fault happens, knowing that this fault occurs within the next $R$ units of time. Replacing $T$ by $R$ in Equation (17), we obtain $\mathbb{E}\left(R_{\text {lost }}\right)=\frac{1}{\lambda}-\frac{R}{e^{\lambda R}-1}$. The expression for $\mathbb{E}\left(\mathrm{TIME}_{\mathrm{rec}}\right)$ simplifies to

$$
\mathbb{E}\left(\text { TimE }_{\mathrm{rec}}\right)=D e^{\lambda R}+\frac{1}{\lambda}\left(e^{\lambda R}-1\right)
$$


Plugging the values of $\mathbb{E}\left(\mathrm{TIME}_{\text {lost }}\right)$ and $\mathbb{E}\left(\mathrm{TIME}_{\mathrm{rec}}\right)$ into Equation (16) leads to the desired value:

$$
\mathbb{E}(\operatorname{Time}(T-C, C, D, R, \lambda))=e^{\lambda R}\left(\frac{1}{\lambda}+D\right)\left(e^{\lambda T}-1\right)
$$

Proposition 3 is the key to proving that the optimal checkpointing strategy

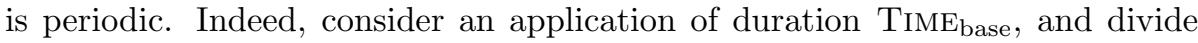
the execution into periods of different lengths $T_{i}$, each with a checkpoint as the end. The expectation of the total execution time is the sum of the expectations of the time needed for each period. Proposition 3 shows that the expected time for a period is a convex function of its length, hence all periods must be equal and $T_{i}=T$ for all $i$.

There remains to find the best number of periods, or equivalently, the size of each work chunk before checkpointing. With $k$ periods of length $T=\frac{\text { TIME }_{\text {base }} \text {, }}{k}$ we have to minimize a function that depends on $k$. This is easy for a skilled mathematician who knows the Lambert function $\mathbb{L}$ (defined as $\mathbb{L}(z) e^{\mathbb{L}(z)}=z$ ). She would find the optimal rational value $k_{\text {opt }}$ of $k$ by differentiation, prove that the objective function is convex, and conclude that the optimal value is either $\left\lfloor k_{o p t}\right\rfloor$ or $\left\lceil k_{o p t}\right\rceil$, thereby determining the optimal period $T_{\text {opt }}$. What if you are not a skilled mathematician? No problem, simply use $T_{\mathrm{FO}}$ as a firstorder approximation, and be comforted that the first-order terms in the Taylor expansion of $T_{\mathrm{opt}}$ is $\ldots T_{\mathrm{FO}}$ ! See [4] for all details.

\section{Appendix 3: MTBF of a platform with $N$ parallel processors}

In this section we give another proof of Proposition 1. Interestingly, it applies to any continuous probability distribution with bounded (nonzero) expectation, not just Exponential laws.

First we prove that Equation (1) does hold true. Consider a single processor, say processor $P_{q}$. Let $X_{i}, i \geq 0$ denote the IID (independent and identically distributed) random variables for the fault inter-arrival times on $P_{q}$, and assume that $X_{i} \sim D_{X}$, where $D_{X}$ is a continuous probability distribution with bounded (nonzero) expectation $\mu_{\text {ind }}$. In particular, $\mathbb{E}\left(X_{i}\right)=\mu_{\text {ind }}$ for all $i$. Consider a fixed time bound $F$. Let $n_{q}(F)$ be the number of faults on $P_{q}$ until time $F$. More precisely, the $\left(n_{q}(F)-1\right)$-th fault is the last one to happen strictly before time $F$, and the $n_{q}(F)$-th fault is the first to happen at time $F$ or after. By definition of $n_{q}(F)$, we have

$$
\sum_{i=1}^{n_{q}(F)-1} X_{i} \leq F \leq \sum_{i=1}^{n_{q}(F)} X_{i} .
$$

$\mathrm{RR} \mathrm{n}^{\circ} 8971$ 
Using Wald's equation [19, p. 420], with $n_{q}(F)$ as a stopping criterion, we derive:

$$
\left(\mathbb{E}\left(n_{q}(F)\right)-1\right) \mu_{\text {ind }} \leq F \leq \mathbb{E}\left(n_{q}(F)\right) \mu_{\text {ind }},
$$

and we obtain:

$$
\lim _{F \rightarrow+\infty} \frac{\mathbb{E}\left(n_{q}(F)\right)}{F}=\frac{1}{\mu_{\text {ind }}} .
$$

As promised, Equation (18) is exactly Equation (1).

Now consider a platform with $N$ identical processors, whose fault interarrival times are IID random variables that follow the distribution $D_{X}$. Unfortunately, if $D_{X}$ is not an Exponential law, then the inter-arrival times of the faults of the whole platform, i.e., of the super-processor of Section 3, are no longer IID. The minimum trick used in the proof of Proposition 1 works only for the first fault. For the following ones, we need to remember the history of the previous faults, and things get too complicated. However, we could still define the MTBF $\mu$ of the super-processor using Equation (18), $\mu$ must satisfy:

$$
\lim _{F \rightarrow+\infty} \frac{\left.\mathbb{E}\left(n_{(} F\right)\right)}{F}=\frac{1}{\mu},
$$

where $\left.n_{(} F\right)$ be the number of faults on the super-processor until time $F$. But does the limit always exist? and if yes, what is its value?

The answer to both questions is not difficult. Let $Y_{i}, i \geq 1$ denote the random variables for fault inter-arrival times on the super-processor. Let $\mu$, with $\mathbb{E}\left(Y_{i}\right)=\mu$. Consider a fixed time bound $F$ as before. Let $n(F)$ be the number of faults on the whole platform until time $F$, and let $m_{q}(F)$ be the number of these faults that strike component number $q$. Of course we have $n(F)=\sum_{q=1}^{N} m_{q}(F)$. By definition, except for the component hit by the last fault, $m_{q}(F)+1$ is the number of faults on component $q$ until time $F$ is exceeded, hence $n_{q}(F)=m_{q}(F)+1$ (and this number is $m_{q}(F)=n_{q}(F)$ on the component hit by the last fault). From Equation (18) again, we have for each component $q$ :

$$
\lim _{F \rightarrow+\infty} \frac{\mathbb{E}\left(m_{q}(F)\right)}{F}=\frac{1}{\mu_{\text {ind }}} .
$$

Since $n(F)=\sum_{q=1}^{N} m_{q}(F)$, we also have:

$$
\lim _{F \rightarrow+\infty} \frac{\mathbb{E}(n(F))}{F}=\frac{N}{\mu_{\text {ind }}}
$$

which answers both questions at the same time!

\section{Appendix 4: Going further with prediction.}

The discussion on predictions in Section 4 has been kept overly simple. For instance when a fault is predicted, sometimes there is not enough time to take 
proactive actions, because we are already checkpointing. In this case, there is no other choice than ignoring the prediction.

Furthermore, a better strategy should take into account at what point in the period does the prediction occur. After all, there is no reason to always trust the predictor, in particular if it has a bad precision. Intuitively, the later the prediction takes place in the period, the more likely we are inclined to trust the predictor and take proactive actions. This is because the amount of work that we could lose gets larger and larger. On the contrary, if the prediction happens in the beginning of the period, we have to trade-off the probability that the proactive checkpoint may be useless (if we take a proactive action) with the small amount of work that may be lost in the case where a fault would actually happen (if we do not trust the predictor). The optimal approach is to never trust the predictor in the beginning of a period, and to always trust it in the end; the cross-over point $\frac{C_{p}}{p}$ depends on the time to take a proactive checkpoint and on the precision of the predictor. See [2] for details.

Finally, it is more realistic to assume that the predictor cannot give the exact moment where the fault is going to strike, but rather will provide an interval of time, a.k.a. a prediction window. More information can be found in [1].

\section{Appendix 5: Going further with replication.}

There are two natural options "counting" faults. The option chosen in Section 5 is to allow new faults to hit processors that have already been hit. This is the option chosen in [10], who introduced the problem. Another option is to count only faults that hit running processors, and thus effectively kill replica pairs and interrupt the application. This second option may seem more natural as the running processors are the only ones that are important for the application execution. It turns out that both options are almost equivalent, the values of their MNFTI only differ by one [6].

Here a few bibliographical notes. Replication has long been used as a faulttolerance mechanism in distributed systems [14], and in the context of volunteer computing [17]. Replication has recently received attention in the context of HPC (High Performance Computing) applications [20, 23, 9, 10]. While replicating all processors is very expensive, replicating only critical processes, or only a fraction of all processes, is a direction being currently explored under the name partial replication.

Speaking of critical processes, we make a final digression. The de-facto standard to enforce fault-tolerance in critical or embedded systems is Triple Modular Redundancy, or TMR [18]. Computations are triplicated on three different processors, and if their results differ, a voting mechanism is called. TMR is not used to protect from fail-stop faults, but rather to detect and correct errors in the execution of the application. While we all like, say, safe planes protected by TMR, the cost is tremendous: by definition, two thirds of the resources are wasted (and this does not include the overhead of voting when an error is identified).

$\operatorname{RR} n^{\circ} 8971$ 


\section{Appendix 6: Scheduling a linear chain of tasks.}

In this exercise you are asked to help Alice (again). She is still writing her thesis but she does not want to checkpoint at given periods of time. She hates being interrupted in the middle of something because she loses concentration. She now wants to checkpoint only at the end of a chapter. She still has to decide after which chapters it is best to checkpoint.

The difference with the original problem is that the checkpoints can only be taken at given time-steps. If we formulate the problem in a abstract way, we have a linear chain of $n$ tasks (the $n$ chapters in Alice's thesis), $T_{1}, T_{2}, \ldots, T_{n}$. Each task $T_{i}$ has weight $w_{i}$ (the time it takes to write that chapter). The cost to checkpoint after $T_{i}$ is $C_{i}$ and the time to recover from $T_{i}$ is $R_{i}$ (these costs are likely proportional to the chapter length). Faults may strike as before, and their inter-arrival times are IID Exponential laws $\operatorname{Exp}(\lambda)$. We must decide after which tasks to checkpoint, in order to minimize the expectation of the total time.

(p)

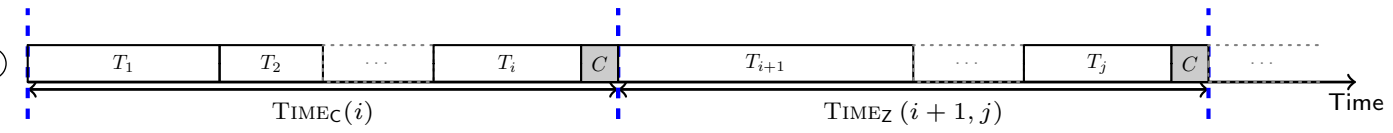

Figure 11: Hint for the exercise.

Figure 11 gives you a hint. $\operatorname{TimE}_{\mathrm{C}}(i)$ is the optimal solution for the execution of tasks $T_{1}, T_{2}, \ldots, T_{i}$. The solution to the problem is $\operatorname{TimE}_{\mathrm{C}}(n)$, and we use a dynamic programming algorithm to compute it. In the algorithm, we need to know Timez $(\mathrm{i}+1, \mathrm{j})$, the expected time to compute a segment of tasks $\left[T_{i+1} . . T_{j}\right]$ and to checkpoint the last one $T_{j}$, knowing that there is a checkpoint before the first one (hence after $T_{i}$ ) and that no intermediate checkpoint is taken. TIMEZ stands for Zero intermediate checkpoint. It turns out that we already know the value of $\operatorname{TimE}_{Z}(i+1, j)$ : check that we have

$$
\operatorname{Time}_{Z}(i+1, j)=\mathbb{E}\left(\operatorname{Time}\left(\sum_{k=i+1}^{j} w_{k}, C_{j}, D, R_{i}, \lambda\right)\right)
$$

and use Proposition 3. 


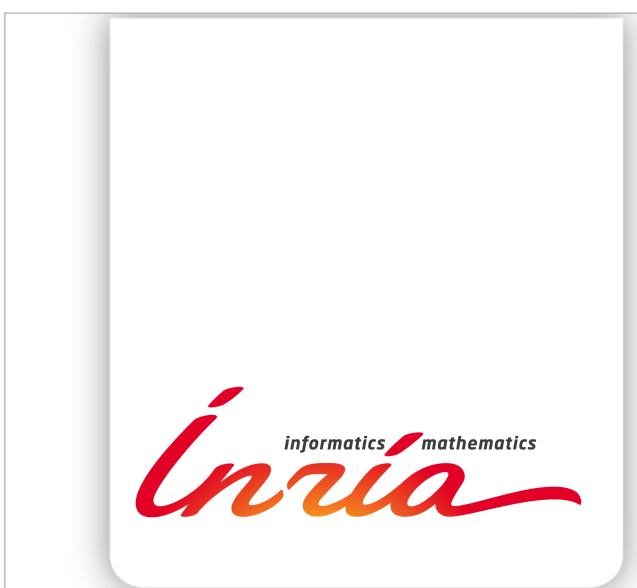

RESEARCH CENTRE

GRENOBLE - RHÔNE-ALPES

Inovallée

655 avenue de l'Europe Montbonnot 38334 Saint Ismier Cedex
Publisher

Inria

Domaine de Voluceau - Rocquencourt

BP 105 - 78153 Le Chesnay Cedex

inria.fr

ISSN 0249-6399 